\title{
Experimental Study on Mechanism of Water-Alternating-Gas Injection in thick Sandstone Reservoir.
}

\author{
Xiaofei $\mathrm{Li}^{1, *}$, Aifen $\mathrm{Li}^{2}$, Wanjiang $\mathrm{Guo}^{2}$, Shiqi $\mathrm{Liu}^{2}$ and Shiti Cui ${ }^{2}$ \\ ${ }^{1}$ School of Petroleum Engineering, China University of Petroleum (East China), Qingdao, China \\ ${ }^{2}$ Exploration and Development Research Institute, Petro China Tarim Oilfield Company, Xinjiang, China
}

\begin{abstract}
For the thick sandstone reservoir, due to gravity differentiation, water drive mainly uses the bottom oil of the reservoir, while gas drive mainly drives the oil along the top of the reservoir. Water-alternating gas (WAG) injection can effectively combine the advantages of water flooding and gas injection, so that gas and water can work in synergy, thus further expanding swept volume and enhancing oil recovery. Over the past half century, the technology has been successfully applied to more than 60 oilfields worldwide, but its mechanism remains to be further studied. In this paper, a total of three tests were conducted through conventional water-flooding (WF), water flooding followed by gas flooding and WAG in sand-packed 2dmodel. These experimental processes are recorded by video with time so that the saturation distribution of each phase in porous media can be observed, migration law of gas and water is studied, and the mechanism of WAG displacement is analyzed. The results show that gravity and capillary force have great influence on the process of gas water alternation, thus increasing the overall swept volume of water and gas; The water/gas alternating injection has the highest oil recovery factor (RF) of $75.45 \%$ in test 3 , in comparison with water flooding followed by gas flooding (70.85\% in test 2$)$ and water-flooding $(66.7 \%$ in test 1$)$; Increase in cycles of WAG tends to reduce residual oil saturation.
\end{abstract}

\section{Introduction}

Alternate gas-water injection, consisting of two traditional water flooding and gas flooding enhanced oil recovery (EOR) techniques, is a promising method for secondary and tertiary recovery.1-5 Water flooding, the volumetric sweep efficiency is relatively high but the microscopic displacement efficiency is low; gas flooding, the microscopic displacement efficiency is relatively high but the volumetric sweep efficiency is low. When gas and water are alternately injected into the reservoir, the gas seepage ability is stronger than that of water. Under the action of pressure, the gas can enter into some small pore oil-bearing channels which are difficult for water to enter. The remaining part of gas and water not only occupy the pore space originally occupied by oil, making oil flow into the low seepage resistance layer, but also redistributes oil, gas and water in the reservoir. At the same time, due to the interference of gas and water phases, the relative permeability of gas and water phases is reduced, and the oil recovery is improved.6-13 In this way, the advantages of water flooding and gas flooding can be combined by sweeping oil-bearing channels with different pore sizes by gas and water flooding, which can not only increase the macro sweep volume, but also improve the micro displacement efficiency.14-17 In addition, it can well control the fluidity of the leading edge, inhibit viscous fingering, and make the leading edge displacement stable, so as to improve sweep efficiency. 18-21
This technology was first used in North Pembina oil field in Alberta, Canada in 1957.22 In 1962, Seelington was the first to apply gas water simultaneous injection in the field practice. 23-24 By the 21st century, gas water alternate injection has become a mature technology in foreign countries and has been successfully applied in many oil fields. The application of gas water alternate injection technology is mainly concentrated in the United States $(63 \%)$, Canada $(15 \%)$ and the former Soviet Union. According to statistics, the incremental oil recovery of WAG is from $5 \%$ to $25 \%$ approximately. 25

However, due to many factors, it is a very complex process to simulate the three-phase flow in the process of alternating gas and water. So far, scholars have done a lot of experimental and theoretical research on the mechanism of gas water alternate injection to enhance oil recovery, but the understanding is not deep enough. In this paper, the mechanism of enhancing oil recovery by gas-water alternation is obtained by the experiment of 2$\mathrm{D}$ plate sand filling model. It is of great significance for the development of thick sandstone reservoirs.

\section{Experiments and methods}

\subsection{Model fabrication}

The sand-packed 2d-model for experiment was composed of perspex sheet and cemented quartz sand. The perspex sheet was bonded into a frame by an acrylic adhesive. The

\footnotetext{
Corresponding author: 1239037300@qq.com
} 
quartz sand and binder were mixed and filled inside the perspex frame in the proportion of $5: 1$, and then compacted and encapsulated. The filling medium is oilwet with a permeability of $1058 \mathrm{mD}$. The model size is $50 \times 10 \times 0.5 \mathrm{~cm}$, see Fig. 1 .

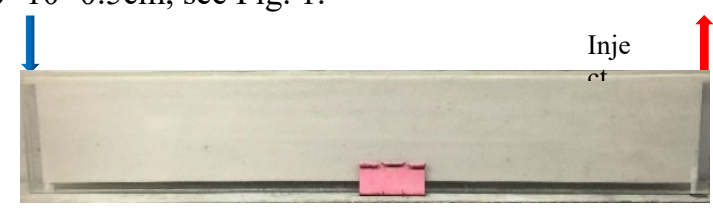

Figure 1. Sand-packed 2d-model

\subsection{Experiment condition}

All the flooding experiments were performed at atmospheric pressure of $1.01 \times 105 \mathrm{~Pa}$ and temperature of $25^{\circ} \mathrm{C}$. The oil in the experiment is aviation kerosene with viscosity of $2.2 \mathrm{mPa} \bullet \mathrm{s}$ and density of $0.794 \mathrm{~g} / \mathrm{cm} 3$. Sudan red was added to the oil to make it appear red. The injected water in the test is distilled water, which is stained with methyl blue, the viscosity $0.894 \mathrm{~m} \mathrm{~Pa} \cdot \mathrm{s}$ and the density $0.997 \mathrm{~g} / \mathrm{cm} 3$. The injection gas is nitrogen supplied by the cylinder. It is colorless. The viscosity is $0.011 \mathrm{mPa} \bullet \mathrm{s}$ and the density is $0.0069 \mathrm{~g} / \mathrm{cm} 3$.

\subsection{The experimental steps}

(1) Established the experimental process according to Figure 3, and checked the leakage of the process. If there was a loss, it should be made up in time according to the cause of the loss before the start of the experiment. (2) In the experiment, the effective pore volume of the model would be determined first.

Weighed the dry weight of the model; The model would be vacuumed for 4 hours; Saturated with simulated oil: The oil displacement rate was kept at $0.2 \mathrm{ml} / \mathrm{min}$, when the oil production rate at the exit was consistent with the oil injection rate, slowly increasing the injection rate to 1 $\mathrm{ml} / \mathrm{min}$ to fully saturate model with oil; And the wet weight of the model was weighed, calculated the volume of saturated oil. (3) Water flooding, water flooding followed by gas flooding and water / gas alternate flooding were carried out respectively, and the distribution of oil, gas and water was recorded in real time by camera. The oil and water separator was used to record the amount of oil produced, and the gas produced was recorded by gas flowmeter. Water cut was calculated according to water production divided by the liquid production. (4) Stopped pumping and completed the experiment until the producing well was no longer producing oil.

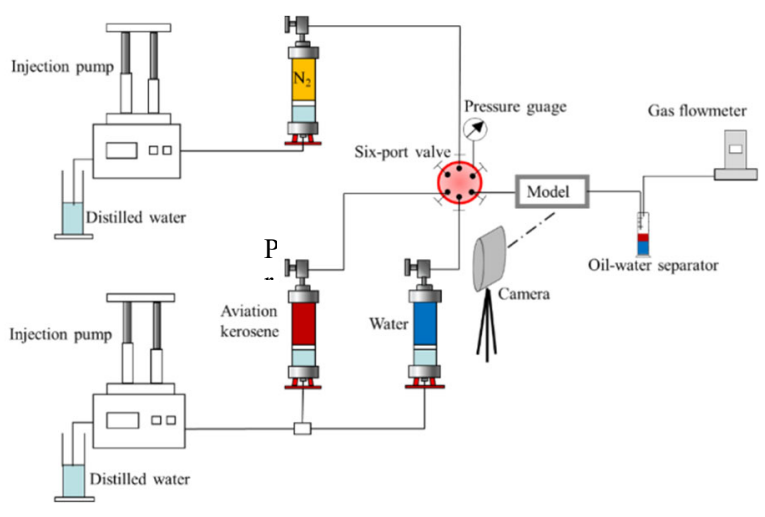

Figure 2. Flow chart of Water-Alternating-Gas Injection. In this work, three different flooding schemes were applied to study the mechanism of WAG displacement in the sandstone formation, i.e., water flooding, Water flooding followed by gas flooding, and water/gas alternating displacement.

The detailed experimental data are listed in Table1.

Table 1. Parameter and Conditions of Experiments.

\begin{tabular}{cccc}
\hline $\begin{array}{c}\text { The serial } \\
\text { number }\end{array}$ & Tests & $\begin{array}{c}\text { Injection } \\
\text { rate }\end{array}$ & $\begin{array}{c}\text { Slug } \\
\text { Size }\end{array}$ \\
\hline 1 & $\begin{array}{c}\text { Water flooding } \\
\text { Water flooding } \\
\text { followed by gas } \\
\text { flooding } \\
\text { Water/gas } \\
\text { alternating } \\
\text { experiment }\end{array}$ & $1.5 \mathrm{~mL} / \mathrm{min}$ & - \\
& $1.5 \mathrm{~mL} / \mathrm{min}$ & - \\
3 & & & \\
& & & \\
\hline
\end{tabular}

\section{Results and discussion}

\subsection{Water flooding experiment}

In water displacing oil (test 1), the oil recovery of continuous water injection was mainly determined by the layer located in the lower part. With the increase of the injected water, the swept area was expanding, it flowed towards the outlet of the model. When the water broke through, a water channel was formed in the model along which the re-injected water preferentially went across the model. Ultimately, a large amount of residual oil was still left behind after WF is applied. (see Figure 3).

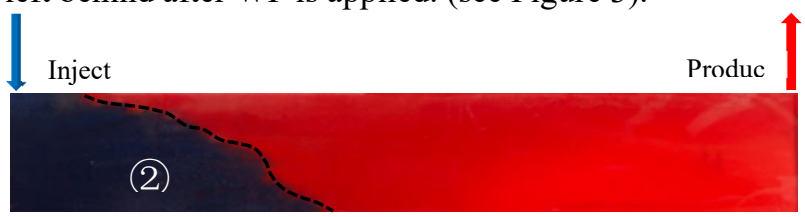

Fluid saturation distribution when water injection volume is $0.34 \mathrm{PV}$.

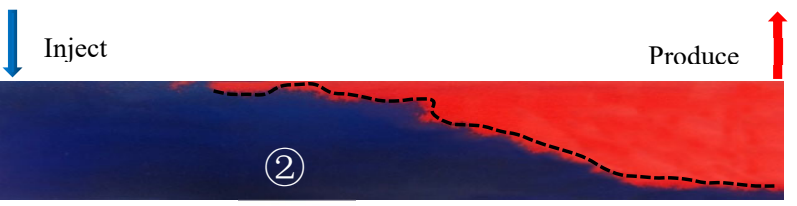

Fluid saturation distribution at the end of Water flooding (1)pure oil region (2)oil-water region

Figure 3. Fluid saturation distribution during the Water flooding. 


\subsection{Water flooding followed by gas flooding}

Water flooding followed by gas flooding (test 2), as depicted in Figure 5, was observed to have higher areal sweep efficiency than water flooding. After water injection, the displacement process mainly occurred in the middle and lower layers of the model. In the ensuing N2 injection processes, gas and water worked together to use oil. In addition, because the gas density was much less than that of water, oil and water were separated under the action of gravity, and the gas used the oil at the top of the upper layer. At that point, there were four flow zones: pure oil region, oil-water region, gas-water region, oil gas and water three-phase region. More oil was displaced as the gas migrates. Gradually, an air channel appeared at the top of the model. After the gas broke through, the oil production decreased obviously.

In gas displacing oil, gas not only drove the upper oil which was not contacted by water, but enlarged the area of water flooding, making the oil-water front moved slightly forward, so that the remaining oil could be produced. (see Figure 4).

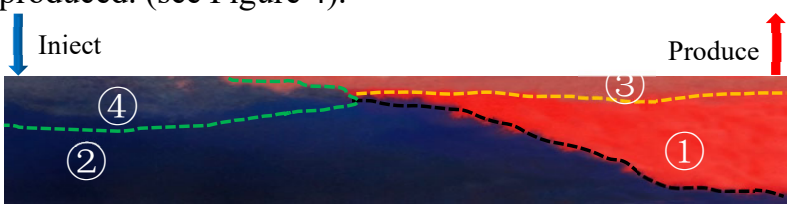

(1)pure oil region (2)oil-water region (3)gas-water region (4)oil-gas-water three-phase region.

Figure 4. Fluid saturation distribution at the end of Water flooding followed by gas flooding.

Figure 5 reveals the relationship between recovery, water cut, and the PV of injected fluids in the Water flooding followed by gas flooding experiment. It is can be seen that, at the initial stage of water injection, the crude oil RF is almost linear with the injected water PV, up to $0.68 \mathrm{pv}$. In this rapid oil recovery period, the injected water replaced the crude oil in the two-dimensional flat plate model (almost equal volume replacement). After water broke through, water and oil were produced together, the oil production rate began to decline, and the water cut increased rapidly. However, there were still a small amount of recovery $(5 \%)$ at the water-cut rising stage. With the increase of amount of water, the injection water has a stronger drag and wash effect on the oil film on the inner wall of the model, and the oil volume decreases. Therefore, there was still a small amount of recovery during the water cut rise stage. At $0.78 \mathrm{PV}$, the model produced water completely, and water drive no longer yielded oil. The ultimate recovery of water injection was $66.7 \%$. The subsequent nitrogen flooding can be roughly divided into two liquid production stages. At the beginning, water and oil were produced at the same time, the oil production rate was lower than that of water drive, and the water cut decreased significantly. In the second stage, after the gas broke through (0.97PV), oil, gas and water were produced at the same time, the oil production rate was greatly reduced, and the water cut increased. Till $1.21 \mathrm{PV}$, and the gas drive no longer produce oil. The final recovery rate was $70.85 \%$, which was $4.15 \%$ higher than that of water flooding.

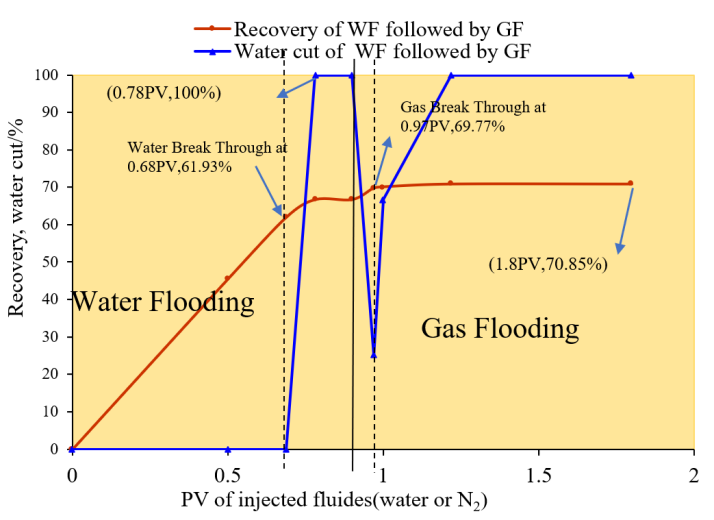

Figure 5 reveals the relationship between recovery

Water/gas alternating experiment

In the WF of the first WAG cycle, the residual oil in the middle and lower part of the model was obviously reduced, as shown in Figure 6. In the GF of the first WAG cycle, the gas drove the water forward and flowed along the upper layer of the model, forming the new oil displacement channels. This is because in the oil wet model, the seepage resistance of the gas in the oil channel is greater than that in the water channel, the injected gas preferentially enters the water channel and pushes the water forward. Then, the gas was separated upward, the injected gas flowed along the upper part of the model to displace the remaining oil under capillary force and buoyancy, as shown in Figure 7.

In the WF of the second WAG cycle, with the injection of water, the oil-water interface moved forward, and the water and oil invaded the gas channel upward under the capillary force. The gas channel was gradually occupied by oil and water, and the longitudinal and transverse swept volume of water expanded at the same time, so as to reduce the remaining oil, as shown in Figure 8. In the GF of the second WAG cycle, the gas entered the model, to establish gas channel and to use more residual oil, as shown in Figure 9.

The following gas water alternation process repeated the above displacement mode. The oil-water interface moved forward continuously, the gas went forward along the upper part, and the gas water transverse sweep area enlarged continuously. The fluctuation of water gas interface and oil gas interface made the volume of water gas longitudinal wave increase. In addition, the threephase region was larger and larger. Finally, the displacement channels of gas and water reached the expansion limit and continued to alternate without oil production, as shown in Figure 10.

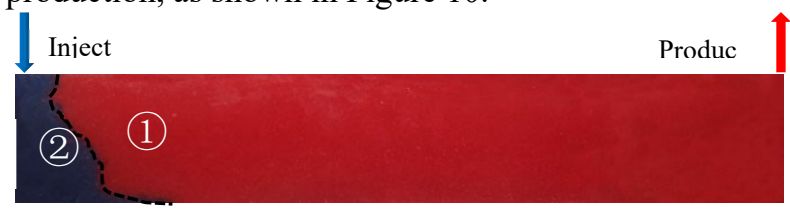

Figure 6. Fluid saturation distribution at first water flooding of WAG 


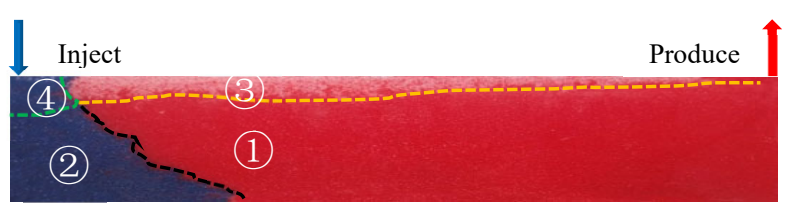

Figure 7. Fluid saturation distribution at first water and gas flooding of WAG

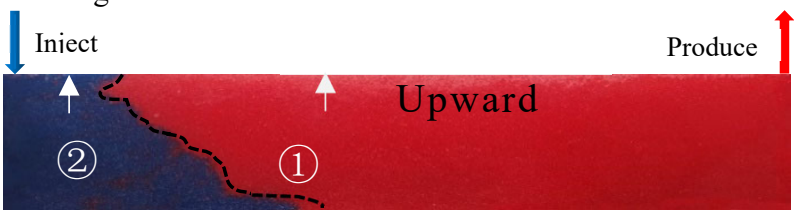

Figure 8. Fluid saturation distribution at second water flooding of WAG

Inject

Produce

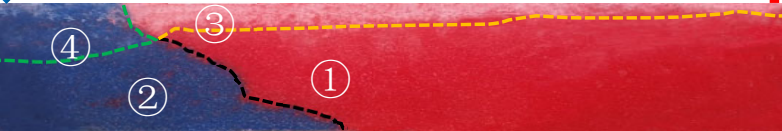

Figure 9. Fluid saturation distribution at second water and gas flooding of WAG

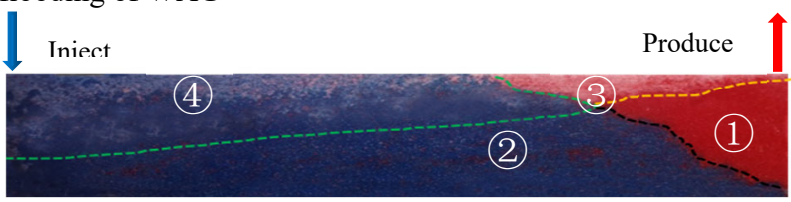

Figure 10. Fluid saturation distribution at the end of WAG displacement

Therefore, through the above experiment, we can clearly understand the gas-water alternation process in the thick sandstone reservoir. As shown in Figure 1, in the process of water gas alternation, the injected water pushes the crude oil forward and downward under the effect of displacement pressure and gravity, mainly contacts the middle and lower parts of the formation, and enters the reservoir macro pores to displace the crude oil, forming a water-oil two-phase zone; Under the displacement pressure, the injected gas migrates forward and downward, invades the oil-water two-phase zone, and works with water to contribute to the oil-gas-water three-phase zone, which greatly improves the displacement efficiency; In addition, due to the large difference of gas and water density, the gas is separated upward, and intrudes into the oil phase area under the action of capillary force and buoyancy, forming the gas-oil two-phase zone. In the end, the pure oil area not affected by gas and water is left.

With the injection of gas and water, the three-phase region of oil-gas-water expands, the oil-water interface advances forward, and the oil invades the oil-gas two-phase region under the action of capillary force, which makes the circulating injected gas displace the crude oil in the same position repeatedly.

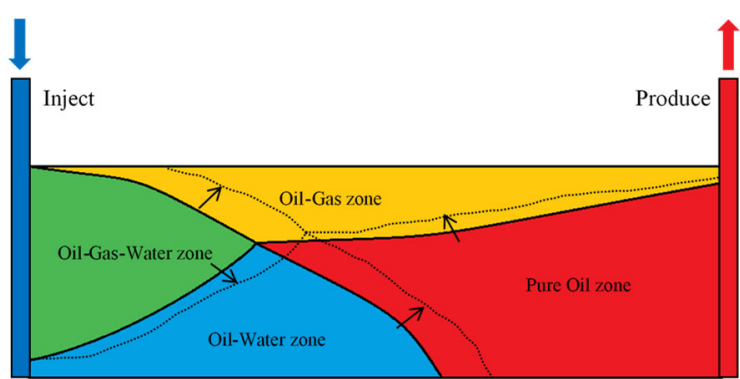

Figure 11. Schematic representation of WAG injection. The relationship between recovery efficiency, water cut and the PV of injected fluids in WAG mode is plotted in Figure 12. The WAG injection can be roughly divided into three production stages. At first, only oil was produced before gas breakthrough, an increased PV of the displacing phase (i.e., the water and N2) led to an increased oil recovery. Specifically, from 0 to $0.2 \mathrm{PV}$, the recovery increased almost linearly; Nevertheless, from 0.2 to $0.23 \mathrm{PV}$ (at the beginning of the second WAG cycle), the oil production rate decreased significantly. It was speculated that the water entering the model mainly drove the crude oil to gas channel, discharging gas to the outlet of model. In the second stage, after gas breakthrough and before water breakthrough, the products were oil and gas. It is worth noting that, generally, after the gas breakthrough, the oil production rate should be greatly reduced, but in this experiment, the oil production rate increased. This was because the gas breakthrough occurred in the early stage of water drive of the second WAG cycle. After the gas was discharged by WF, water continued to drive the crude oil, therefore, a amount of the residual oil was further recovered after N2 breakthrough. Finally, after water breakthrough, oil, gas and water were produced simultaneously. Compared with the previous production, the overall oil production rate declined significantly. The oil production rate was reduced gradually and the final oil RF reached a maximum value at $2 \mathrm{PV}$. It is found that the water cut does not increase monotonically, dropping at 1.4 PV and 1.8 PV. This was because gas re-entered the pores and effectively displaced crude oil, resulting in higher oil recovery than during the water-injection phase of the same cycle.

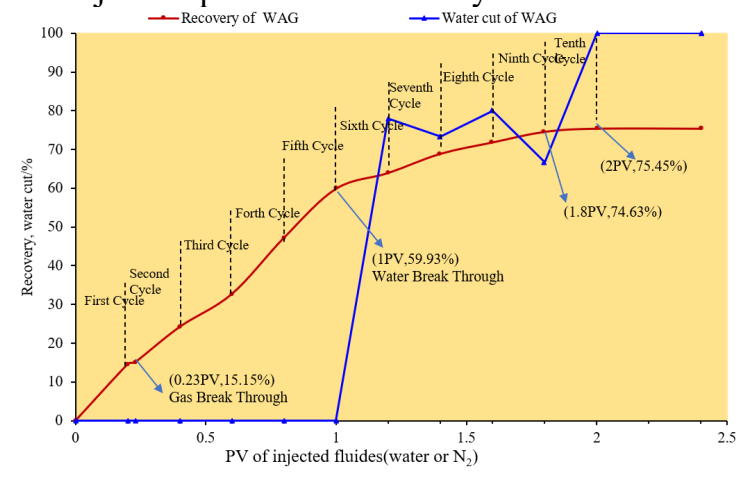

Figure 12. Oil recovery and water cut versus PV of injected fluid in the water/gas alternating experiment.

Obviously, compared with figure 13 and figure 6, when the injected fluid PV is 1.8 , the recovery of WAG is higher 
than that of Water flooding followed by gas flooding. Hence, the number of WAG cycles has a strong effect on the oil Recovery, if the injected fluid volume is fixed, the more WAG cycles (that is, the smaller the size of gas water alternation slug), the higher the oil recovery. Compared with pure water flooding, water flooding followed by gas flooding and WAG, it is seen that WAG has the highest oil RF. The increase in recovery becomes smaller and smaller as the number of WAG cycles increase (see Fig13)

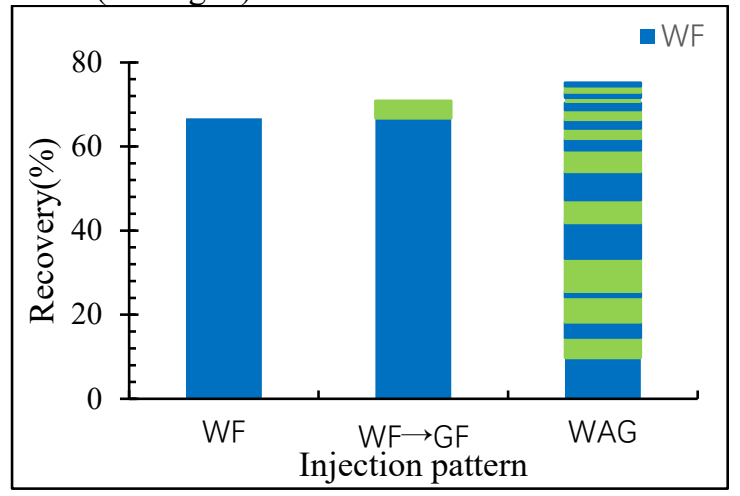

Figure 13. Oil recovery under different injection patterns.

\section{Conclusion}

(1) The WAG injection process overcame the disadvantages of small sweep volume and large residual oil volume by only water displacement, and gave the better sweep control, mobility control of water, and the recovery factor was obviously better than that of water flooding. Therefore, now the application of WAG injection methods is more and more widely.

(2) Gravity and capillary force played an important role in the alternating process. Due to the gravity differentiation, water and gas acted on the bottom and upper layer of the model respectively to displace together; Due to the existence of capillary force, water and oil intruded into the gas drive area, and gas displaced crude oil at the same location for many times to improve the displacement efficiency.

(3) The effect of multiple WAG was better than that of single WAG. The more the alternation cycles were, the higher the efficiency was.

\section{Acknowledgments}

This work was financially supported by Key Science and Technology Program of Petro China. (ZD2019-183-008).

\section{References}

1. Sohrabi, M.; Tehrani, D. H.; Danesh, A.; Henderson, G. D., Visualization of Oil Recovery by WaterAlternating-Gas Injection Using High-Pressure Micromodels. SPE Journal2004, 9, (03), 290-301.

2. Kulkarni, M. M.; Rao, D. N., Experimental investigation of miscible and immiscible WaterAlternating-Gas (WAG) process performance.
Journal of Petroleum Science and Engineering2005, 48, (1-2), 1-20.

3. Christensen, J. R.; Stenby, E. H.; Skauge, A., Review of WAG field experience. Presented at the International Petroleum Conference and Exhibition of Mexico, Villahermose, Mexico, March 3-5, 1998; Paper SPE 39883.

4. Li, Z.; Yin, Y.; Wang, Q., Research progress on mechanism of enhanced oil recovery by water alternating gas injection. Journal of Southwest Petroleum University2007, 2, 2226, 180

5. Guo, P.; Du, Z.; Zhang, M. , Study on the development of water alternating gas injection hydrocarbon miscible flooding in Pubei oilfield. Journal of Southwestern Petroleum In- stitute2004, 4, (25-27), 37-90.

6. Christensen, J. R.; Stenby, E. H.; Skauge, A. Review of WAG Field Experience. SPE Res. Eval. Eng. 2001, 4 (2), 97-106. Paper SPE 71203.

7. Surguchev, L. M.; Korbol, R.; Krakstad, O.S.,Optimum Water Alternate Gas Injection Schemes for Stratified Reservoir. Presented at the Annual Technical Conference and Exhibition, Washington, DC, October 4-7, 1992. Paper SPE 24646.

8. Tiffin, D. L.; Yelling, W. F., Effects of Mobile Water on Multiple-Contact Gas Displacements. SPEJ1983, June, 447.

9. John, D. R., A Literature Analysis of the WAG Injectivity Abnormalities in the $\mathrm{CO} 2$ Process. SPE Reservoir Evaluation \& Engineering: 2001.

10. Guzman, R. E., Three-Phase Flow in Field-Scale Simulation of Gas and WAG Injections. Presented at the SPE European Petroleum Conference, London, October 25-27, 1994; Paper SPE 28897.

11. Han, L.; Gu, Y. Optimization of Miscible CO 2WAG Injection in the Bakken Formation. Energy Fuels 2014, 28 (11), 6811-6819.

12. Fatemi, S. M.; Sohrabi, M. Recovery Mechanisms and Relative Permeability for Gas/Oil Systems at Near-Miscible Conditions: Effects of Immobile Water Saturation, Wettability, Hysteresis, and Permeability. Energy Fuels 2013, 27 (5), 2376-2389.

13. Godec, M. L.; Kuuskraa, V. A.; Dipietro, P. Opportunities for Using Anthropogenic CO2 for Enhanced Oil Recovery and CO 2 Storage. Energy Fuels 2013, 27 (8), 4183-4189.

14. Christensen, J. R.; Larse, M.; Nicolaisen, H., Compositional simulation of water-alternating-gas processes. Presented at the Annual Technical Conference and Exhibition, Dallas, Texas, USA, October 1-4, 2000; Paper SPE 62999.

15. Aleidan, A. A.; Mamora, D. D. SWACO2 and WACO2 Efficiency Improvement in Carbonate Cores by Lowering Water Salinity. Presented at the SPE Canadian Unconventional Resources and International Petroleum Conference, Calgary, Alberta, October 19-21, 2010; Paper SPE 137548. 
16. Shetty, S.; Hughes, R. G.; Afonja, G. Experimental Evaluation of Simultaneous Water and Gas Injection using Carbon Dioxide. Presented at the SPE EOR Conference at Oil and Gas West Asia, Muscat, Oman, March 31-April 2, 2014 ; Paper SPE 169690.

17. 17. Kulkarni, M. M.; Rao, D. N. Experimental Investigation of Miscible and Immiscible WaterAlternating-Gas (WAG) Process Performance. J. Pet. Sci. Eng. 2005, 48 (1), 1-20.

18. Nezhad, S. A. T., Mojarad, M. R. R., Moghadas, J. S., Experimental study on applicability of wateralternating-CO2 injection in the secondary and tertiary recovery. SPE 103988: 2006.

19. 19. Chen, S; Li, H.; Yang, D., Optimal parametric design for water alternating-gas (WAG) process in a CO2-miscible flooding reservoir. Journal of Canadian Petroleum Technology2010, 49,(10),7582.

20. Ramachandran, K. P.; Gyani, O. N.; Sur, S., Immiscible hydrocarbon WAG: Laboratory to field. SPE 128848: 2010.

21. 21. Taheri, A.; Sajjadian, V. A., WAG performance in a low porosity and low permeability reservoir. SPE 100212: 2006.

22. Du, J.; Liu, W.; Guo, P., Study on variation rule of water-alternating-gas injection capacity in low permeability reservoirs. Journal of Southwest Petroleum University ( Natural Science Edition)2011, 33, (5), 114-117, 198 .

23. 23. Zhang, Z., Alternate injection of water and gas to improve recovery efficiency. Journal of Daxian Teachers College2002, (2),33-35.

24. 24. Walker, J. W.; Turner, J. L., Performance of Seeligson Zone 20B-07enriched- gas- drive project. Journal of Petroleum Technology 1968, 20, (4), 369373.

25. 25. Koottungal, Leena, SPECIAL REPORT: EOR/Heavy Oil Survey: 2010 worldwide EOR survey, Oil and Gas Journal, April 19, 2010. 